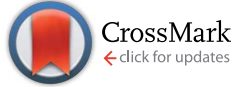

Cite this: RSC Adv., 2017, 7, 10609
Received 21st December 2016 Accepted 1st February 2017

DOI: $10.1039 / c 6 r a 28509 k$

rsc.li/rsc-advances

\section{Solvent-based delignification and decrystallization of wheat straw for efficient enzymatic hydrolysis of cellulose and ethanol production with low cellulase loadings $\dagger$}

\author{
Tian Li,,$^{\mathrm{a}}$ Qi Fang,,$^{\mathrm{a}}$ Hongmei Chen, ${ }^{\mathrm{a}}$ Feng Qi, ${ }^{\mathrm{c}}$ Xianjin Ou, ${ }^{\mathrm{d}}$ Xuebing Zhao*ab \\ and Dehua Liu ${ }^{\text {ab }}$
}

Several solvents and ionic liquids, namely formic acid (Formiline process), concentrated phosphoric acid (CPA), $\mathrm{N}$-methylmorpholine- $\mathrm{N}$-oxide (NMMO) and 1-allyl-3-methylimidazolium chloride ([AMIM]Cl), were used to pretreat wheat straw in order to increase cellulose digestibility for ethanol production. When being directly used to pretreat the raw wheat straw under corresponding optimized conditions, the improvement of cellulose hydrolyzability followed the order of CPA > Formiline $>\mathrm{NMMO}>[\mathrm{AMIM}] \mathrm{Cl}$. However, when Formiline pretreated (delignified) substrates were further post-treated by the above cellulose solvents, the cellulose digestibility was significantly improved particularly with low cellulase loadings. Cellulose solvent post-treatment resulted in deconstruction of hydrogen-bond networking, alteration of cellulose polymorphs, decrease in crystallinity, depolymerization of cellulose chain with dramatic reduction in particle size, thus greatly increasing cellulose accessible surface area. CPA posttreatment showed the best efficacy. Semi-simultaneous scarification and fermentation (sSSF) of a CPA post-treated substrate obtained an ethanol concentration of $41.6 \mathrm{~g} \mathrm{~L}^{-1}$ with $91.2 \%$ of yield at a relatively low cellulase loading (5 FPU per g solid) within $24 \mathrm{~h}$ incubation. A biorefining process was proposed based on Formiline pretreatment coupled with CPA post-treatment to achieve a co-production of ethanol, furfural and high-purity lignin, which greatly increases the potential revenue of the process.

\section{Introduction}

Lignocellulosic biomass has been considered as one of the most promising non-food feedstocks for producing secondgeneration bioethanol. However, release of fermentable sugars from lignocellulosic biomass has become a limiting step for efficient and economical production of ethanol from cellulose, mainly due to the biomass recalcitrance constructed by chemical components, cell wall structure as well as

\footnotetext{
${ }^{a}$ Institute of Applied Chemistry, Department of Chemical Engineering, Tsinghua University, Beijing 100084, China. E-mail: zhaoxb@mail.tsinghua.edu.cn; Fax: +8610-62772130; Tel: $+86-10-62772130$

${ }^{b}$ Tsinghua Innovation Center in Dongguan, Dongguan 523808, China

${ }^{c}$ College of Life Sciences, Engineering Research Center of Industrial Microbiology, Fujian Normal University, Fuzhou 350108, China

${ }^{d}$ Institute of Biophysics, Chinese Academy of Sciences, Beijing 100101, China

$\dagger$ Electronic supplementary information (ESI) available: Comparison of $24 \mathrm{~h}$ enzymatic hydrolysis of different solvent post-treated substrates; correlations between hydrogen-bond intensity and enzymatic glucan conversion with different cellulase loadings; photomicrographs of pretreated substrates with adsorption of fusion protein probe molecules; and conceptual design of biorefining of lignocellulosic biomass based on solvent treatments. See DOI: $10.1039 / \mathrm{c} 6 \mathrm{ra} 28509 \mathrm{k}$

$\$$ These authors contribute equally to this work.
}

cellulose supramolecular structure. ${ }^{1}$ One of the most important factors limiting cellulose accessibility for enzymatic hydrolysis is the presence of lignin, which binds the cells, fibers and vessels like a "glue" to reinforce cell walls and keep them from collapsing. Lignin is covalently bound to the side chains of branched hemicelluloses to form a lignin-carbohydrate complex (LCC), which has been found to be an important factor to biomass recalcitrance. ${ }^{2}$ Lignin also can irreversibly adsorb cellulase enzymes, reducing the efficient cellulase concentration in the liquid phase and productive contact between cellulose substrate and enzymes. ${ }^{3,4}$ Therefore, removing lignin has been found to dramatically increase cellulose digestibility. ${ }^{5}$ Another structural factor limiting enzymatic hydrolysis of cellulose is the crystallinity. ${ }^{6}$ Cellulose contains amorphous and crystalline structures. In amorphous region, the cellulose is present in a less order form because that the hydrogen bond network is not well established. Therefore, amorphous cellulose is much less recalcitrant to enzymatic hydrolysis. In crystalline region, cellulose chains are present in high order due to the hydrogen bond network, so that crystalline cellulose is much more. Therefore, decrystallization of cellulose has been found to greatly accelerate the enzymatic hydrolysis of cellulose., 
Various chemical processes have been developed for removing lignin to increase cellulose accessibility, such as alkaline saponification, ${ }^{9}$ oxidative delignification, ${ }^{10}$ organic solvent extraction ${ }^{11}$ and sulfite pulping. ${ }^{12,13}$ Among these pretreatments, the solvent (organosolv)-based process not only greatly improve cellulose accessibility but also achieve a biomass fractionation to lignin, hemicellulosic sugars, and a relatively pure cellulose fraction, all of which show promises for further production of biofuels and biochemicals in a biorefinery concept. ${ }^{\mathbf{1 1 , 1 4}}$ The used solvent can be easily recovered by simple distillation, not like the complex recovery process for recovery chemicals for alkaline pretreatment (pulping). Among the organic solvents used for delignification, organic acid such as formic acid (FA), shows several merits: (1) it has a Hildebrand solubility parameter $\left(\delta\right.$ value) close to that of lignin $\left(\sim 11 \mathrm{cal}^{1 / 2}\right.$ per $\mathrm{cm}^{-3 / 2}$ ) thus showing a good solvency to lignin fragments; ${ }^{15}$ (2) it can dissociate $\mathrm{H}^{+}$which can accelerate dissolution of hemicellulose and fragmentation of lignin; (3) it has a strong enough acidity thus avoiding using external mineral acid such as $\mathrm{H}_{2} \mathrm{SO}_{4}$ or $\mathrm{HCl} ;{ }^{16}$ and (4) the pretreatment could be conducted under mild condition and atmospheric pressure thus minimizing degradation of pentose. Pretreatment of biomass based on formic acid delignification have been proven to efficiently expose cellulose. ${ }^{16-19}$

Cellulose solvent-based pretreatment also have been found to greatly enhance cellulose hydrolyzability mainly by deconstruction of crystalline structure. ${ }^{20,21}$ One of the most efficient cellulose-solvent is concentrated $\mathrm{H}_{3} \mathrm{PO}_{4}$ (CPA) (>80 wt\%). ${ }^{22}$ It can completely dissolve cellulose fibers, resulting in effective disruption of highly ordered hydrogen bonding network of crystalline cellulose, and the regenerated cellulose is amorphous and very suitable for hydrolysis. ${ }^{23}$ As reported by Zhang et al., ${ }^{24}$ CPA pretreatment resulted in dramatic increase in cellulose digestibility of various lignocellulosic feedstocks with a low cellulase loading. Another efficient cellulose-solvent is ionic liquid (IL), which has attracted much attention in recent years. ${ }^{25}$ In this pretreatment, the oxygen and hydrogen atoms of cellulose hydroxyl groups can form electron donor-electron acceptor complexes interacting with the ILs, and the breaking of hydrogen bonds leads to an opening of the lignocellulose network of linkages, resulting in cellulose dissolution. ${ }^{25}$ Subsequently, using an anti-solvent (e.g., ethanol, acetone, methanol or water), the solubilized cellulose can be quickly precipitated, and the regenerated cellulose has the same or decreased DP compared with the initial cellulose, but significantly different macro- and micro-structure, especially for the degree of crystallinity. ${ }^{25,26}$ However, due to the presence of other components such as lignin, the solubility of biomass in cellulose solvent generally is lower than that of pure cellulose, though lignin also can be dissolved in some of the solvents. ${ }^{27}$ Therefore, if a great part of lignin can be removed prior to cellulose dissolution by cellulose solvents, the pretreatment efficiency might be further improved in terms of additionally improving cellulose digestibility, increasing cellulose recovery and decreasing the amount of cellulose solvent used. Therefore, the objective of this work is to compare the efficiency of several solvent-based pretreatments to improve cellulose hydrolyzability as well as fermentability of wheat straw (WS). Particularly, a FA based pretreatment (Formiline process) developed in our group ${ }^{16,17}$ was compared and coupled with CPA or IL treatments, in order to achieve a high cellulose conversion with relatively low cellulase loadings.

\section{Experimental}

\section{Materials}

The WS used in the experiments was collected from Shandong Province, China. It consisted of 35.1\% glucan (cellulose), 23.4\% xylan, $21.1 \%$ total lignin, $6.8 \%$ ash and $13.6 \%$ others. It was chopped to about $1 \mathrm{~cm}$ long prior to pretreatment. All the chemicals used in the experiments, mainly including formic acid (88 wt\%, FA), phosphoric acid (85 wt\%), acetone, ethanol, glacial acetic acid, calcium hydroxide, dimethylsulfoxide (DMSO), methyl gallate, $N$-methylmorpholine- $N$-oxide (NMMO) (50 wt\%), 1-methylimidazole and chloropropene were purchased locally. The standard chemicals for HPLC including cellobiose, glucose and xylose were purchased from SigmaAldrich (Shanghai branch, China). NMMO was concentrated to $84 \%$ by vacuum evaporation before use, since only concentrated NMMO can dissolve cellulose. ${ }^{28}$ Ionic liquid, 1-allyl-3methylimidazolium chloride ([AMIM]Cl) was prepared according to ref. 29. The cellulase formula used was Cellic ${ }^{\circledR}$ CTec2, which was kindly provided by Novozymes (Beijing branch, $\mathrm{CN}$ ).

\section{Solvent pretreatment and post-treatment}

The Formiline pretreatment comprises a FA delignification (FAD) with 80 wt\% FA and an alkaline deformylation (AD) with a small amount of alkali as developed and optimized in our previous work. ${ }^{16}$ The FAD was carried out in a $1000 \mathrm{~mL}$ threeneck glass flask heated by electric jacket under atmospheric pressure. $30 \mathrm{~g}$ screened WS was packed into the flask followed by addition of $300 \mathrm{~mL} 80 \mathrm{wt} \%$ FA solution. Electrical stirring with a Teflon paddle was used at $300 \mathrm{rpm}$ for keeping the system as homogeneous as possible. The delignification was controlled at the boiling point of the FA solutions under atmospheric pressure $\left(\sim 107^{\circ} \mathrm{C}\right)$ for $1 \mathrm{~h}$. After delignification, the mixture was filtered under vacuum. The obtained solid was first washed with $300 \mathrm{~mL} 80 \mathrm{wt} \%$ FA solution and then filtered to remove as much liquid as possible. Typically, after the vacuum filtration the liquid content of the pretreated solid was $75-80 \%$. The obtained solid was then washed thoroughly by water till neutrality. For the $\mathrm{AD}$ process, the water-washed solid was mixed thoroughly with $2 \mathrm{wt} \% \mathrm{Ca}(\mathrm{OH})_{2}$ (based on initial WS weight) and heated to $120{ }^{\circ} \mathrm{C}$ for $1 \mathrm{~h}$ in an autoclave to remove the formyl group introduced during FAD process. The deformylated solid was then washed with water for further enzymatic hydrolysis or analysis.

CPA pretreatment was performed according to the optimized procedure reported by Sathitsuksanoh et al. ${ }^{20}$ with some modification. $10 \mathrm{~g}$ of chopped WS was placed in a $500 \mathrm{~mL}$ round-bottom flask followed by addition of $200 \mathrm{~g} 85 \mathrm{wt} \%$ CPA. The flask was then placed in a $50{ }^{\circ} \mathrm{C}$ water bath for $1.5 \mathrm{~h}$. The reaction mixture was stirred with a Teflon paddle at $300 \mathrm{rpm}$. 
After reaction, $400 \mathrm{~g} 4{ }^{\circ} \mathrm{C} 95 \mathrm{wt} \%$ ethanol was immediately added as an anti-solvent to the reaction mixture. The solid was filtered with a 160-mesh Nylon cloth and filter paper and thoroughly washed by water to neutrality, and stored at $4{ }^{\circ} \mathrm{C}$ for further enzymatic hydrolysis and chemical composition analysis.

[AMIM]Cl pretreatment of WS was according to the optimized work of Zhang et al. ${ }^{29} 10 \mathrm{~g}$ of chopped WS was pretreated by $200 \mathrm{~g}$ [AMIM]Cl in a $500 \mathrm{~mL}$ round-bottom flask stirred at $300 \mathrm{rpm}, 110^{\circ} \mathrm{C}$ for $1 \mathrm{~h}$. After the reaction system was cooled to below $100{ }^{\circ} \mathrm{C}, 400 \mathrm{~g}$ boiling water was added as an anti-solvent to the reaction mixture. The solid was then filtered and washed by water to neutrality.

NMMO pretreatment of WS was according to the optimized work of Kuo and Lee. ${ }^{30} 10 \mathrm{~g}$ of chopped WS was pretreated by $200 \mathrm{~g}$ NMMO and $1 \mathrm{~g}$ methyl gallate as an antioxidant in a 500 $\mathrm{mL}$ round-bottom flask stirred at $300 \mathrm{rpm}, 130^{\circ} \mathrm{C}$ for $2 \mathrm{~h}$. After the reaction system was cooled to below $100{ }^{\circ} \mathrm{C}, 400 \mathrm{~g}$ boiling water was added as an anti-solvent to the reaction mixture. The solid was then filtered and washed by water to neutrality.

The solvent post-treatments were performed with the same procedures of corresponding solvent pretreatments, but with acetone-dried Formiline pretreated solid as feedstock instead of raw WS. To decrease the viscosity of [AMIM]Cl-cellulose system, [AMIM]CL/DMSO with a molar ratio of $7: 3$ was also used for post-treatment according to Tian et al.,$^{31}$ and other posttreatment condition was similar to that for [AMIM]Cl posttreatment. Since direct oven-drying may lead to change of structural features of the pretreated substrates, solvent such as acetone was used to wash the substrates for several times prior to lyophilization in a freezing-drier for subsequent characterization of the pretreated cellulosic solid.

\section{Enzymatic hydrolysis of pretreated substrates}

The enzymatic hydrolysis were performed with cellulase loading of $0.75-15$ FPU per $g$ solid at $50 \pm 0.5{ }^{\circ} \mathrm{C}$ and $\mathrm{pH} 4.8(0.1 \mathrm{M}$ sodium acetate buffer) in an air-bath shaker at $130 \mathrm{rpm}$ for 5 days with initial solid loading of $1-10 \%\left(\mathrm{~g} \mathrm{~mL}^{-1}\right)$. The enzymatic digestibility was described as enzymatic glucan conversion (EGC) defined as follows:

$$
\begin{aligned}
& \text { Enzymatic glucan conversion }(\mathrm{EGC}, \%)= \\
& \qquad \frac{\left(w_{\text {glucose }}+w_{\text {cellobiose }}\right) \times 0.9}{w_{\mathrm{IG}}} \times 100 \%
\end{aligned}
$$

where $w_{\text {glucose }}$ and $w_{\text {cellobiose }}$ are the weight of glucose and cellobiose produced by enzymatic hydrolysis; $w_{\text {IG }}$ was the weight of glucan in pretreated solid. Sugar concentrations in the hydrolyzate were determined by HPLC, and glucan conversions were calculated according to sugar concentrations and liquid column. Each reported datum was the average of at least duplicate tests.

\section{Semi-simultaneous saccharification and fermentation}

Semi-simultaneous scarification and fermentation (sSSF) was carried out in $25 \mathrm{~mL}$ Erlenmeyer flasks with total working volume of $10 \mathrm{~mL}$ with an air shaker incubator at $150 \mathrm{rpm}$ with
5-15\% (w/v, $\left.\mathrm{g} \mathrm{mL}^{-1}\right)$ solid substrate loading. Other nutrients including $2 \mathrm{~g} \mathrm{~L}^{-1}\left(\mathrm{NH}_{4}\right)_{2} \mathrm{SO}_{4}, 5 \mathrm{~g} \mathrm{~L}^{-1} \mathrm{KH}_{2} \mathrm{PO}_{4}, 5 \mathrm{~g} \mathrm{~L}^{-1}$ yeast extract, $1 \mathrm{~g} \mathrm{~L}^{-1} \mathrm{MgSO}_{4}$ and $0.2 \mathrm{~g} \mathrm{~L}^{-1} \mathrm{CaCl}_{2}$ dissolved in $\mathrm{pH} 5.3$ $\mathrm{H}_{2} \mathrm{SO}_{4}$ solution was then added. The mixture was autoclaving at $121{ }^{\circ} \mathrm{C}$ for $20 \mathrm{~min}$ for sterilization. After the mixture was cooled down to room temperature, cellulase enzymes of 5 and 15 FPU per $\mathrm{g}$ solid were added. The mixture was then incubated at $50{ }^{\circ} \mathrm{C}$ for pre-hydrolysis for $24 \mathrm{~h}$ followed by inoculation of yeast Saccharomyces cerevisiae (Angel Yeast Co., Ltd, China). Samples were taken every 6, 12 or $24 \mathrm{~h}$ for analyzing ethanol and sugar concentrations. The ethanol yield was calculated according to ethanol concentration and expressed as the percentage of theoretical maximum ethanol yield.

\section{Analytic methods}

The main chemical compositions of raw WS and pretreated substrates were determined according to NREL's Laboratory Analytical Procedure. ${ }^{32}$ Monosaccharides concentrations were determined by Shimadzu (Tokyo, Japan) HPLC (LC-10AT) system as described in our previous work. ${ }^{33}$ Cellulase, xylanase and $\beta$-glucosidase activities were determined according to the procedures described by Zhao et al. ${ }^{34}$

Fourier transform infrared spectroscopy (FTIR) spectra of the acetone dried samples were recorded with a NICOLET 560 FTIR spectrometer (Nicolet Company, USA). The acetone-dried samples were embedded in $\mathrm{KBr}$ pellets with an approximate concentration of $1 \mathrm{mg} / 100 \mathrm{mg} \mathrm{KBr}$. The spectra were recorded in the range of $4000-400 \mathrm{~cm}^{-1}$.

Crystallinity of the acetone-dried pretreated substrates was determined by X-ray diffraction (XRD) using D8 ADVANCE diffractometer (Bruker AXS, Germany). The diffractogram was taken by $\theta-2 \theta$ method. Samples were scanned at $1^{\circ} \mathrm{min}^{-1}$ from $2 \theta=5-50^{\circ}$ with a step size of $0.01^{\circ}$. The crystallinity was determined as the percentage of crystalline material in the sample and expressed as the crystallinity index (CrI):

$$
\mathrm{CrI}=\left[\left(I_{002}-I_{\mathrm{am}}\right) / I_{002}\right] \times 100
$$

where $I_{002}$ is the maximum intensity of the (002) lattice diffraction $\left(2 \theta \approx 22.5^{\circ}\right)$ and $I_{\mathrm{am}}$ is the intensity for the amorphous scattering. It should be noted that cellulose I and II showed different diffraction patterns, and the region for the intensity of the amorphous fraction was somewhat different, $2 \theta$ $=\sim 18.7^{\circ}$ for cellulose I and $2 \theta=\sim 16^{\circ}$ for cellulose II. ${ }^{35}$

The surface morphology of raw WS and acetone-washed pretreated solids was performed using scanning electron microscope (SEM, HRSEM JSM7401, JEOL, Japan). Prior to imaging, the samples were sputter-coated with a thin layer of gold to make the fibers conductive, avoiding degradation and buildup of charge on the specimen. Images were obtained at magnifications ranging of $\times 300, \times 3000$ and $\times 30000$.

\section{Results and discussion}

\section{Direct pretreatment of wheat straw with different solvents}

WS was directly pretreated by different solvents under corresponding optimal conditions reported in literatures. As shown 
Table 1 Chemical compositions of substrates pretreated by different solvents

\begin{tabular}{|c|c|c|c|c|c|c|c|}
\hline Solvent & $G_{\ln }{ }^{a}(\%)$ & $X_{\ln ^{b}}^{b}(\%)$ & $L_{\mathrm{ig}}{ }^{c}(\%)$ & $\mathrm{SY}^{d}(\%)$ & $R_{\mathrm{Gln}}^{e}(\%)$ & $S_{\mathrm{X} \ln }^{f}(\%)$ & $\mathrm{DD}^{g}(\%)$ \\
\hline FA (Formiline) & 75.5 & 6.2 & 8.30 & 40.7 & 87.5 & 89.2 & 84.0 \\
\hline CPA & 63.5 & 4.2 & 22.2 & 41.1 & 73.7 & 91.9 & 52.5 \\
\hline NMMO & 39.1 & 28.9 & 11.5 & 73.2 & 81.5 & 9.6 & 60.1 \\
\hline
\end{tabular}

${ }^{a} G_{\mathrm{ln}}$ : glucan content. ${ }^{b} X_{\mathrm{ln}}$ : xylan content. ${ }^{c} L_{\mathrm{ig}}$ : lignin content. ${ }^{d}$ SY: solid yield based on initial dry solid weight. ${ }^{e} R_{\mathrm{Gln}}$ : recovery of glucan based on initial glucan weight. ${ }^{f} S_{\mathrm{XIn}}$ : solubilization of xylan based on initial xylan weight. ${ }^{g}$ DD: degree of delignification based on initial lignin weight.

in Table 1, FA (Formiline pretreatment) achieved the highest degree of delignification (DD, defined as the percentage of lignin removed) and glucan recovery $\left(R_{\mathrm{Gln}}\right)$, indicating that it has the highest delignification selectivity to avoid glucan dissolution in the pretreatment. However, Formiline pretreatment also dissolved a great fraction of xylan with a low solid yield (SY, defined as the percentage of solid weight recovered after pretreatment or post-treatment). Nevertheless, the glucan content in the pretreated solid $\left(G_{\ln }\right)$ greatly increased compared to that of raw WS due to removal of lignin and xylan. CPA pretreatment resulted in a considerable dissolution of xylan and a moderate DD. However, the $R_{\mathrm{Gln}}$ was relatively lower than those of other solvent pretreatments. Wang et al. ${ }^{36}$ found that after CPA pretreatment of various biomass feedstocks, the cellulose recovery generally was in the range of $60-90 \%$, while hemicellulose removal in the range of $78-100 \%$. The relatively low $R_{\mathrm{Gln}}$ of CPA pretreatment might be because CPA could cause a significant depolymerization of glucan and xylan, and thus a part of the dissolved polysaccharide many present as soluble oligosaccharide, which could be recovered as solid phase. Moreover, the presence of hemicelluloses and lignin degradation products in the system might exert some negative impacts on the precipitation of dissolved cellulose from the CPA solution. The glucan contents of [AMIM]Cl and NMMO pretreated samples were relatively lower, and corresponding $R_{\mathrm{Gln}} \mathrm{S}$ were similar to that of Formiline pretreatment. However, the solubilizations of xylan $\left(S_{\mathrm{XIn}} \mathrm{s}\right)$ was much lower than those of Formiline and CPA pretreatment. Moderate DDs were obtained by these two solvent pretreatments.

The time courses of enzymatic hydrolysis of different solvent-pretreated wheat straw at 5\% solid loading with different cellulase loadings are shown in Fig. 1. Cellulase loading showed a significant effect on the EGC of Formiline pretreated substrates (Fig. 1A). For example, within $24 \mathrm{~h}$ enzymatic incubation, the EGCs were 10.30, 36.0, 54.6 and $81.9 \%$ at cellulase loadings of $0.75,3.4,7.5$ and 15 FPU per $g$ solid, respectively. For a long-time ( $120 \mathrm{~h})$ hydrolysis, the EGCs were $20.8,53.2,89.1$ and $96.8 \%$, respectively, indicating that a relatively high cellulase loading was needed for Formilinepretreated substrates to achieve a high cellulose conversion. CPA pretreatment demonstrated a dramatically improved cellulose digestibility. EGC could reach $83.5 \%$ within $12 \mathrm{~h}$ hydrolysis with a cellulase loading of $3.5 \mathrm{FPU}$ per g solid. The EGC even reached $80 \%$ at $120 \mathrm{~h}$ with a very low cellulase loading (0.75 FPU per g solid), while higher than $95 \%$ with cellulase loading of $\geq 3.5 \mathrm{FPU}$ per $\mathrm{g}$ solid. [AMIM]Cl and NMMO pretreatments showed much poorer enzymatic digestibility (Fig. 1C and D). EGCs@120 h were just 35.3 and 64.7\%, respectively, with cellulase loading of 15 FPU per $g$ solid, respectively. This was mainly because WS had a much complex structure than pure cellulose and [AMIM]Cl and NMMO pretreatments could not completely dissolve the biomass. Moreover, $[\mathrm{AMIM}] \mathrm{Cl}$ and NMMO pretreatments still retained a great part of hemicelluloses and lignin in the pretreated substrates, which limited the cellulose accessibility.

\section{Post-treatment of Formiline-pretreated wheat straw with different cellulose solvents}

As found in the above experimental results, lignocellulosic biomass is much more complicated in structure than pure cellulose, and its solubilization in cellulose solvent would be more difficult. Particularly, lignin binds the cells as a "glue" to prevent the permeation of chemical agents. If lignin was removed prior to solvent treatment for decrystallization, the cellulose digestibility would be more efficiently improved. Formiline pretreatment could achieve a good removal of lignin with liberation of cellulose fibers. ${ }^{18}$ Therefore, Formiline pretreated solid was further post-treated by different cellulose solvents. As shown in Table 2, all of the solvents just removed a small fraction of lignin, and showed high SYs with glucan
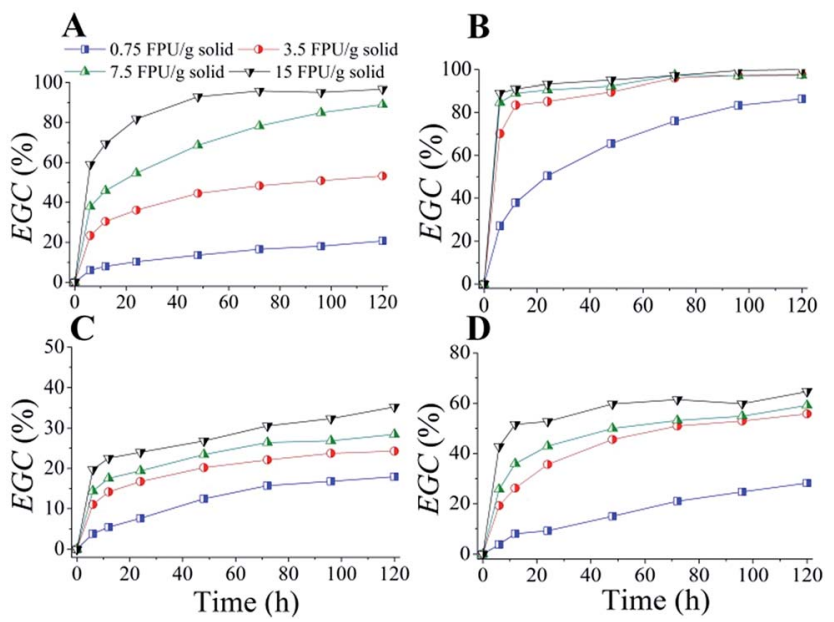

Fig. 1 Enzymatic hydrolysis of different solvent-pretreated wheat straw at $5 \%$ solid loading with different cellulase loadings. (A) Formiline pretreatment; (B) CPA pretreatment; (C) [AMIM]Cl pretreatment; (D) NMMO pretreatment. 
Table 2 Chemical compositions of substrates obtained by solvent post-treatment of Formiline pretreated wheat straw

\begin{tabular}{lllllll}
\hline Post-treatment & $G_{\ln }{ }^{a}(\%)$ & $X_{\ln }{ }^{b}(\%)$ & $L_{\mathrm{ig}}{ }^{c}(\%)$ & $\mathrm{SY}^{d}(\%)$ & $R_{\mathrm{Gln}}{ }^{e}(\%)$ & $S_{\mathrm{Xln}}{ }^{f}(\%)$ \\
\hline CPA & 80.4 & 2.04 & 8.23 & 91.6 & 97.5 & 69.9 \\
[AMIM]Cl & 73.6 & 5.39 & 8.06 & 96.2 & 93.8 & 16.3 \\
[AMIM]Cl/DMSO & 73.0 & 5.31 & 8.35 & 93.3 & 90.2 & 9.2 \\
NMMO & 80.6 & 4.97 & 9.16 & 88.1 & 94.0 & 20.1 \\
\hline
\end{tabular}

${ }^{a} G_{\mathrm{In}}$ : glucan content. ${ }^{b} X_{\mathrm{In}}$ : xylan content. ${ }^{c} L_{\mathrm{ig}}$ : lignin content. ${ }^{d}$ SY: solid yield based on Formiline-pretreated dry solid. ${ }^{e} R_{\mathrm{Gln}}$ : recovery of glucan based on glucan weight of Formiline-pretreated dry solid. ${ }^{f} S_{\mathrm{Xln}}$ : solubilization of xylan based on xylan weight of Formiline-pretreated dry solid.

${ }^{g}$ DD: degree of delignification based on lignin weight of Formiline-pretreated dry solid.

recovery higher than $90 \%$. CPA post-treatment further removed about $70 \%$ of the residual xylan of Formiline-pretreated solid, while ionic liquid [AMIM]Cl showed the poorest ability for xylan solubilization.

The time courses of enzymatic hydrolysis of the post-treated cellulosic substrates at 5\% solid loading (Fig. 2) demonstrated that solvent post-treatment indeed were efficient to improve cellulose digestibility of Formiline-pretreated substrates, especially with low cellulase loadings. At cellulase loading of 0.75 FPU per $\mathrm{g}$ solid, the EGC@120 increased from $20.7 \%$ for Formiline pretreatment to 56.5, 39.2, 25.58, and 30.3\% for CPA, [AMIM]Cl, [AMIM]Cl/DMSO and NMMO post-treatments, respectively (Fig. 2A). CPA post-treatment achieved the best cellulose digestibility. EGC could reach about $80 \%$ within $24 \mathrm{~h}$ hydrolysis with 3.5 FPU per $\mathrm{g}$ solid of cellulase, compared with $36.3 \%$ for samples without post-treatment and 50.1, 42.1 and $43.5 \%$ for substrates post-treated by [AMIM]Cl, [AMIM]Cl/ DMSO and NMMO, respectively (Fig. 2B). At a high cellulase loading such as 15 FPU per $g$ solid, the difference of the EGCs among these post-treated substrates became less significant. About $90 \%$ conversion could be obtained for all of the samples after $120 \mathrm{~h}$ hydrolysis (Fig. 2D).

Effects of solid loading on EGC@24 h were further compared as shown in ESI Fig. S1. $\dagger$ These results further clearly
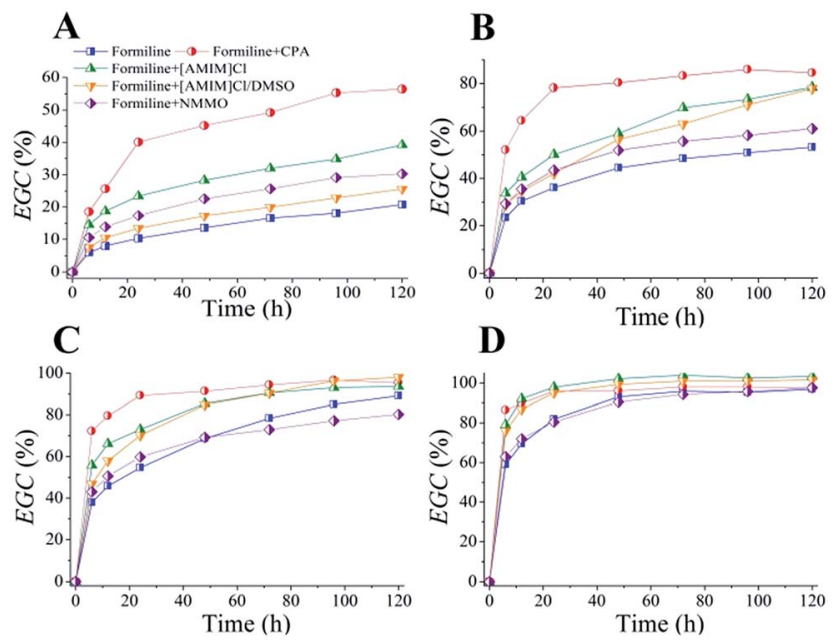

D

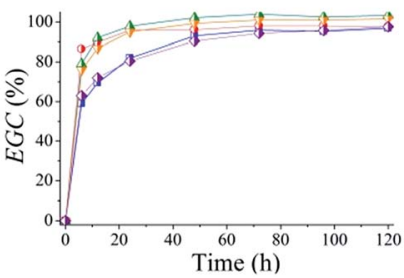

Fig. 2 Enzymatic hydrolysis of different solvent post-pretreated substrates after Formiline pretreatment at 5\% solid loading with different cellulase loadings. (A) 0.75 FPU per g solid; (B) 3.75 FPU per g solid; (C) 7.5 FPU per g solid; (D) 15 FPU per g solid. corroborated that solvent post-treatment greatly improved cellulose digestibility. Except CPA post-treated sample, which showed the highest EGC at solid loading of 5\%, other samples generally showed somewhat decreased EGCs with increase in solid loading from $1-10 \%$. This is because that solid loading greatly influences the system viscosity, rheology and mass transfer, especially at high-solid system, thus accordingly significant affect cellulose hydrolysis. ${ }^{37}$ The change of EGC versus solid loading was also dependent on post-treatment process. For example, with cellulase loading of 3.5 FPU per $g$ solid, CPA post-treatment obtained EGCs of 74.16, 78.2 and $71.6 \%$ at solid loading of 1,5 , and $10 \%$, respectively; while [AMIM]Cl obtained EGCs of 57.2, 50.1 and 39.3\%, respectively. With a high cellulase loading such as 15 FPU per g solid, solid loading showed no significant impacts on CPA, [AMIM]Cl and [AMIM]Cl/DMSO post-treated substrates, while it still apparently affected the EGCs of NMMO-pretreated samples $(90.7,80.4$ and $77.1 \%$ at 1,5 and $10 \%$ solid loading, respectively). It indicated the enzymatic hydrolysis condition also showed significant interaction with the structural features of the substrates.

\section{Structural features of post-treated substrates}

The structural features of Formiline pretreated and solvent post-treated substrates were further characterized by photography and SEM as shown in Fig. 3. The raw WS had much larger size (photograph) and clear beehive-like cell wall structure at cross section (SEM image with 300 magnifications) with compact, non-porous and smooth surface (SEM image with 1000 and 30000 magnifications). Different cell types such as

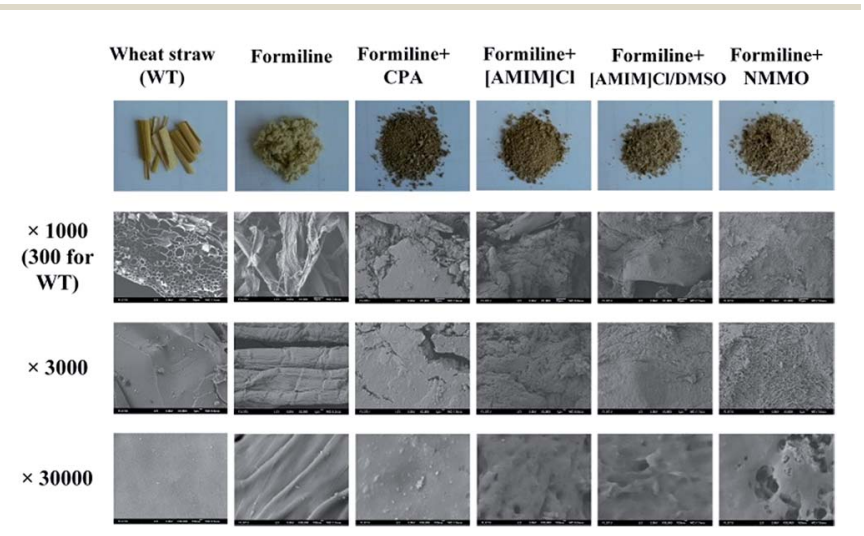

Fig. 3 Photographs and SEM images of raw wheat straw and substrates pretreated by different solvents. 
epidermis cells, parenchyma cells, vascular bundles as well as thick-walled fiber cells can be seen, as observed by Kristensen et $a{ }^{38}$ This structure definitely is recalcitrant to enzymatic hydrolysis. Microscopically, such a recalcitrant structure, just like a world of reinforced concrete, is constructed by the cell wall compositions. Cellulose fibers act as the rebars or steel rods to provide strength over long distances. Hemicellulose represents the wire mesh or cable that wraps around the celluloses rods, providing extra strength and linkages. Lignin acts as the "concrete" that fills the remaining gaps and sets, holding everything in place and excludes water from the polysaccharide environment. ${ }^{39}$ Formiline pretreatment dissolved a considerable fraction of hemicelluloses and lignin, liberating cellulose fibers, thus the pretreated solid was pulp-like. This was because formic acid as an organic acid could hydrolyze hemicellulose and fragmentize lignin macromolecule. The lignin fragments are then dissolved in aqueous formic acid, thus achieving a high degree of delignification. ${ }^{\mathbf{1 8 4 0}}$ This process is the same as that of formic acid pulping, which causes cellulose fiber liberation from cell wall. Therefore, clear fiber was observed by SEM, but lignin aggregate that usually can be seen for thermochemical pretreatment, ${ }^{41}$ was not observed on the fiber surface due to the mild pretreatment condition. After solvent post-treatment, the pulp-like substrates became much finer, looking like powders in dry state (photographs) while gellike in wet state. No fiber was observed by SEM images. The substrates also became much rougher with pores. Such porous structure definitely greatly increased the cellulose accessibility.

FTIR spectra (Fig. 4A) analysis indicated that after Formiline pretreatment, the absorption peaks at $\sim 1600$ and $1500 \mathrm{~cm}^{-1}$ disappeared, which were assigned to aromatic skeletal vibrations of lignin, ${ }^{\mathbf{4}}$ indicating that most of lignin was removed by Formiline pretreatment. The bands at $3405 \mathrm{~cm}^{-1}$, which was associated with hydrogen-bonded $\mathrm{O}-\mathrm{H}$ stretching of cellulose, became stronger than that of WS. This was mainly because that most of hemicellulose and lignin were removed by Formiline pretreatment and the cellulose content increased in the pretreated substrates. However, the intensity of this band became weaker after solvent post-treatments, indicating that hydrogenbonding of the cellulose was destroyed to a certain extent. Generally, the bands $4000-2995 \mathrm{~cm}^{-1}, 2900 \mathrm{~cm}^{-1}, 1430 \mathrm{~cm}^{-1}$,
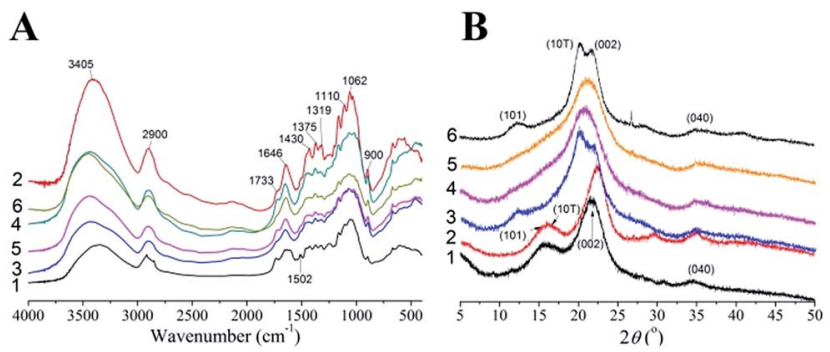

Fig. 4 FTIR spectra (A) and XRD diffractograms (B) of raw wheat straw and substrates pretreated by different solvents: (1) raw wheat straw; (2) Formiline pretreated; (3) Formiline + CPA pretreated; (4) Formiline + [AMIM]Cl pretreated; (5) Formiline + [AMIM]Cl/DMSO pretreated; (6) Formiline + NMMO pretreated.
$1375 \mathrm{~cm}^{-1}$ and $900 \mathrm{~cm}^{-1}$, which are attributed to the $\mathrm{OH}$ stretching, the $\mathrm{CH}$ stretching, the $\mathrm{HCH}$ and $\mathrm{OCH}$ in-plane bending vibrations, the $\mathrm{CH}$ deformation vibration and the COC, CCO, and $\mathrm{CCH}$ deformation and stretching vibrations, respectively, are especially sensitive to the state of the crystalline and amorphous regions. Thus, the relative absorbance ratio ( $\left.A_{4000-2995} / A_{993}\right)$ can be used to represent the hydrogen-bond intensity (HBI). ${ }^{43}$ The HBIs were calculated as 1.792, 1.001, 1.226, 1.318 and 1.569 for Formiline-pretreated, CPA, [AMIM]Cl, [AMIM]Cl/DMSO and NMMO post-treated substrates, respectively. This result further corroborated that solvent posttreatment could destroy the hydrogen-bond networking of cellulose, and CPA was the most effective to deconstruct the crystalline region. HBI showed a significant negative relationship with EGC of the post-treated substrates, especially at low cellulase loading for a short hydrolysis time (see ESI Fig. S2 $\dagger$ ). For example, when substrates were hydrolyzed by 3.5 FPU per $\mathrm{g}$ solid cellulase at $5 \%$ solid loading for $24 \mathrm{~h}$, HBI had a correlation coefficient of -0.85 with EGC@2 4 h. It demonstrated that decrease in HBI is beneficial to improving cellulose digestibility of Formiline pretreated solid.

XRD was further used to study the crystallinity and cellulose polymorphs of post-treated substrates as shown in Fig. 4B. The cellulose of raw WS and Formiline-pretreated substrates were typical cellulose I which has diffraction peaks at $2 \theta=\sim 15.2^{\circ}$ (101 lattice plane), $16.4^{\circ}$ (10 $\overline{1}$ lattice plane), $23^{\circ}$ (002 lattice plane) and $35^{\circ}$ (040 lattice plane). ${ }^{44}$ It indicated that Formiline pretreatment did not alter the polymorph of cellulose. CPA and NMMO post-treated substrates exhibits a typical diffraction pattern of cellulose II which shows a primary $10 \overline{1}$ peak at $20.3^{\circ}$ overlapping with a reduced 002 peak at $21.5^{\circ}$, and a separation between 101 peak at $12.5^{\circ}$ and $10 \overline{1}$ peak. ${ }^{45}$ The [AMIM]Cl and [AMIM]Cl/DMSO showed more different diffraction pattern, where a primary peak at approximately $2 \theta=20.7^{\circ}$ was observed, due to the overlap of the two diffraction planes of (101) and (002). ${ }^{46}$ This diffraction pattern was almost like that of amorphous cellulose. ${ }^{47}$ It was calculated that the CrI was $57.6 \%$ for Formiline-pretreated sample, while CrI decreased to $<30 \%$ for solvent post-treated substrates. For pure cellulose, crystallinity was a key factor limiting the enzymatic hydrolysis rate. Initial hydrolysis rate could be greatly increased by decreasing cellulose crystallinity. This is because a reduction in crystallinity relates to a higher surface area, thus increasing the substrate adsorption capacity to cellulase enzymes. ${ }^{6}$ Moreover, cellulose digestibility is also relevant to the polymorph. As found by Wada et al. ${ }^{48}$ the enzymatic hydrolysis of cellulose I could be greatly accelerated via its conversion to the cellulose II hydrate form. Therefore, the above results indicated that solvent posttreatment resulted in a deconstruction of hydrogen-bond networking with alteration of cellulose polymorph and decrystallization to form amorphous structure, which accordingly significantly improve cellulose digestibility. However, different solvents achieve the decrystallization of cellulose by similar mechanisms. CPA plays a dual role in cellulose dissolution, namely swelling and dissolving the cellulose fiber by effective disruption of highly ordered hydrogen bonding network of crystalline cellulose, and cleaving glycosidic linkage for 
depolymerization. ${ }^{23,49} \mathrm{NMMO}$ dissolves cellulose fibers due to its high polarity $\mathrm{N}-\mathrm{O}$ bond, which breaks the hydrogen bond network of the cellulose and forms new hydrogen bonds with the solute, and the oxidative ability of NMMO also contributes to depolymerization of cellulose. ${ }^{23}$ Cellulose dissolution in ionic liquids is mainly due to the formation of hydrogen bond between cellulose and cations of ionic liquids, causing breaking up the inter- and intramolecular hydrogen bonds in cellulose. ${ }^{\mathbf{5 0}}$ However, the depolymerization of cellulose fiber with dramatic decrease in particle size was also important to increase cellulose accessible surface area, especially for CPA post-treatment. Using a protein probe molecule constructed by fusion of CBM (cellulose binding module) of Trichoderma reesei cellobiohydrolase I with GFP (green fluorescent protein), ${ }^{51}$ which can be specifically adsorbed on cellulose and gives florescent signal for visualizing florescent images and measuring signal intensity for probe concentration (Fig. S3†), we estimated the accessible surface areas (ASAs) of Formiline pretreated and CPA post-treated substrates were 10.8 and $20.4 \mathrm{~m}^{2} \mathrm{~g}^{-1}$, respectively, corroborating that CPA post-treatment indeed exposed more cellulose surface for cellulase binding.

\section{Semi-simultaneous saccharification and fermentation of solvent post-treated substrates for ethanol production}

Formiline pretreated and CPA post-treated substrates were further converted to ethanol by semi-simultaneous saccharification and fermentation (SSSF) with relatively low (5 FPU per $g$ solid) and high (15 FPU per $g$ solid) cellulase loadings at different solid loadings as shown in Fig. 5. CPA post-treatment indeed greatly improved the cellulose digestibility and fermentability, particularly with low cellulose loading. For example, with cellulase of 5 FPU per $\mathrm{g}$ solid, after pre-hydrolysis for $24 \mathrm{~h}$, Formiline pretreatment obtained $48.4,52.3$ and $43.9 \mathrm{~g}$ $\mathrm{L}^{-1}$ glucose concentrations with corresponding 98,54 and $31 \%$
A
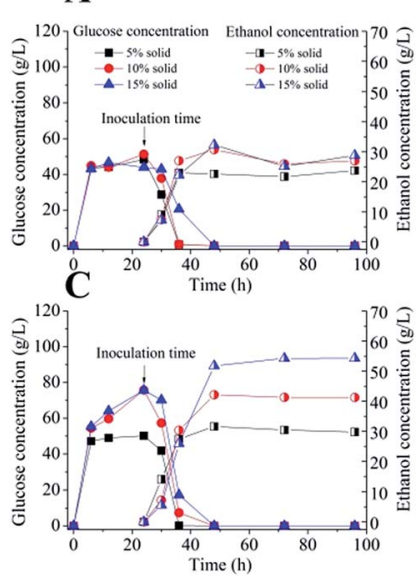

B
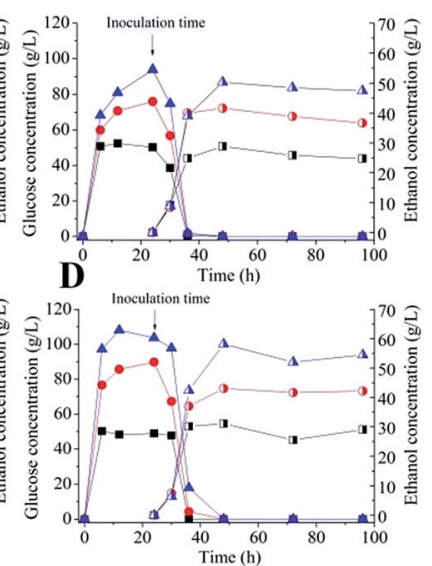

Fig. 5 Semi-simultaneous saccharification and fermentation of pretreated substrates for ethanol production. (A) Formiline-pretreated substrates with 5 FPU per g solid cellulase loading; (B) CPA posttreated substrates with 5 FPU per g solid cellulase loading; (C) Formiline-pretreated substrates with 15 FPU per g solid cellulase loading; (D) CPA post-treated substrates with $15 \mathrm{FPU}$ per $\mathrm{g}$ solid cellulase loading. of cellulose conversions at 5, 10 and 15\% solid loadings, respectively. However, for CPA post-treated substrates, 50.2, 76.0 and $93.8 \mathrm{~g} \mathrm{~L}^{-1}$ glucose concentrations were obtained with $\sim 100 \%, 77$ and $62 \%$ cellulose conversions, respectively. After inoculation with Saccharomyces cerevisiae, glucose concentration quickly decreased to nearly zero with $24 \mathrm{~h}$, indicating that the hydrolyzate could be well converted to ethanol. With 5 FPU per $\mathrm{g}$ solid of cellulase, Formiline pretreated samples obtained 23.0, 30.7 and $32.3 \mathrm{~g} \mathrm{~L}^{-1}$ ethanol with $\sim 100,70.4$ and $49.4 \%$ yields at 5, 10 and 15\% solid loading, respectively, while corresponding ethanol concentrations for CPA post-treated substrates were 28.9, 41.6 and $50.4 \mathrm{~g} \mathrm{~L}^{-1}$ with yields of $\sim 100$, 91.2 and $73.6 \%$, respectively. Increasing cellulase loading to 15 FPU per $g$ solid greatly improved cellulose-to-ethanol conversion for Formiline pretreated substrates, with yields of $94 \%$ at $10 \%$ solid and $83.0 \%$ at $15 \%$ solid, respectively, which were similar to those for CPA post-treated substrates. These results illustrated that CPA post-treatment indeed significantly increase cellulose hydrolyzability with great reduction of enzyme loading to achieve a high cellulose conversion. Cellulase loading could be reduced by $2 / 3$ for CPA post-treated substrates to obtain an ethanol yield of $>90 \%$ compared with Formiline pretreated solid, which would be important to reduce the saccharification cost. It has been found that the enzyme loading and ethanol yield are the main factors affecting the enzyme cost for cellulosic ethanol production. ${ }^{52}$ Enzyme cost accounts for $18.5-62 \%$ of the minimum ethanol selling price (MESP) when cellulase loading increased from 5 to $35 \mathrm{mg}$ protein per $\mathrm{g}$ cellulose, with corresponding MESPs of 2.21-4.71 USD per gal ethanol ${ }^{53}$ based on an actual purchased price of cellulase enzyme in industrial enzyme market. Therefore, CPA post-treatment would make great sense to reduce the enzyme cost.

\section{Conceptual design of the solvent-based treatment for biomass conversion and mass balance}

Based on Formiline pretreatment followed by CPA-post treatment, a process to produce different products from lignocellulosic biomass such as wheat straw can be conceptually designed as shown in Fig. S4. $\dagger$ The Formiline pretreatment can achieve a fractionation of the biomass, by which cellulose is recovered as a solid phase, while most of lignin and hemicelluloses dissolve in the aqueous formic acid. The cellulosic solid is firstly washed by fresh formic acid solution and followed by flashing to recover the residual formic acid. The flashed solid is then post-treated by CPA for decrystallization and depolymerization, and regenerated by using anti-solvent such as ethanol. The regenerated cellulose is then converted to ethanol by SSSF. A part of the spent liquor from pretreatment and washing stage is sent to distillation column for recovering formic acid, while the concentrated spent liquor from the bottom is added with water to precipitate lignin. The liquid phase is then heated to convert xylose into furfural under the catalysis of the residual formic acid. Therefore, by this process, co-production of ethanol, furfural and high-purity lignin can be achieved, which is a demonstration of solvent-based biorefining of lignocellulosic biomass. 


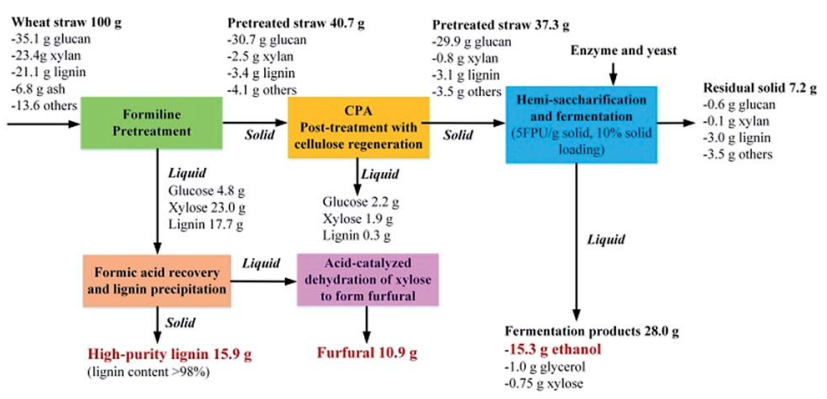

Fig. 6 Mass balance of solvent based fractionating pretreatment for delignification and decrystallization of wheat straw to produce ethanol, furfural and high-purity lignin. The yield of formic acid catalyzed conversion of xylose to furfural was selected as $74 \%$ according to work of Yang et al. ${ }^{58}$

Mass balance (Fig. 6) showed that from $100 \mathrm{~g}$ of wheat straw, $15.3 \mathrm{~g}$ ethanol was produced with co-production of $11.5 \mathrm{~g}$ furfural and $15.9 \mathrm{~g}$ high-purity lignin. Furfural is an important platform chemical, from which a series of derivative chemicals can be produced including some important chemical intermediate such as furfuryl alcohol, furoic acid, furfuryl amine, furanone, furan, methyl furan, maleic acid and so on. ${ }^{54,55}$ The isolated lignin can be further used as a feedstock for producing adsorbents, carbon materials such as activated carbon and carbon fiber, fuels, dispersants, phenolic precursors. Particularly, the lignin product obtained in this process is of highpurity, and thus can be used for glue, binders and polymer substitutions which has a high selling-price. ${ }^{56}$ With lignin and furfural selling prices of 1000 and 1600 USD per $t,{ }^{57}$ and ethanol selling price of 600 USD per $t$, we can estimate that from 1 ton of raw wheat straw ( 80 USD per $\mathrm{t}$ ), the total added-value of the produced products can reach 425 USD per $t$ wheat straw, 4 times increased. However, if the carbohydrates (cellulose and xylan) of the biomass is completely converted to ethanol ( 0.328 ton of ethanol produced from 1 ton of raw wheat straw) with lignin used as a fuel (price of 62 USD per $t$ ), ${ }^{57}$ the maximum revenue would be 210 USD per $t$ wheat straw, only a half of that obtained by the process of this work. Moreover, by CPA post-treatment, the needed cellulase loading could be dramatically reduced to achieve a required ethanol yield. Thus the cost for saccharification would be greatly reduced. However, it should be noted that the decrease in cellulase loading was at the expense of using solvent (FA and CPA), which might oppositely increase the final production cost because of solvent loss and more energy consumption for solvent recovery. These issues might be solved by process design with heat integration. More optimizations and evaluations should be performed by techno-economic analysis.

\section{Conclusions}

Several solvents were used to directly pretreat wheat straw under corresponding optimized conditions in order to improve cellulose hydrolyzability. However, only CPA seems to be efficient to achieve a high $(>80 \%)$ cellulose conversion at low cellulase loading (3.5 FPU per $\mathrm{g}$ solid) within a short incubation time ( $12 \mathrm{~h}$ ), while Formiline pretreatment obtained a cellulose conversion of $89 \%$ at a relatively high cellulase loading (7.5 FPU per $\mathrm{g}$ solid) for a long hydrolysis time $(120 \mathrm{~h})$. However, the CPA pretreatment obtained a relatively low cellulose recovery, and the substrates still had a high lignin content. Therefore, cellulose solvent post-treatment of Formiline pretreated substrates were further investigated in order to reducing cellulose loading for saccharification. The cellulose solvent post-treatment indeed could greatly accelerate the rate of enzymatic hydrolysis of Formiline-pretreated solid using a low cellulase loading, and CPA was the most efficient for the improvement. Cellulose solvent post-treatment resulted in deconstruction of hydrogenbond networking, alteration of cellulose polymorph from cellulose I to II, decrease in crystallinity, depolymerization of cellulose chain with dramatic reduction in particle size, thus greatly increasing cellulose accessible surface area. An ethanol concentration of $41.6 \mathrm{~g} \mathrm{~L}^{-1}$ with $91.2 \%$ yield was obtained for CPA post-treated substrate with $5 \mathrm{FPU}$ per $\mathrm{g}$ solid of cellulase at $10 \%$ solid loading. A mass balance analysis demonstrated that Formiline pretreatment followed by CPA post-treatment for biorefining of lignocellulosic biomass could achieve a much higher potential revenue than that of conventional cellulosic ethanol production process.

\section{Acknowledgements}

This work was supported by National Natural Science Foundation of China (No. 21106081, 21406130), and Dongguan Social and Technical Development Project (No. 2015108101004).

\section{Notes and references}

1 X. Zhao, L. Zhang and D. Liu, Biofuels, Bioprod. Biorefin., 2012, 6, 465.

2 Y. Pu, F. Hu, F. Huang, B. H. Davison and A. J. Ragauskas, Biotechnol. Biofuels, 2013, 6, 15.

3 Y. Zhou, H. Chen, F. Qi, X. Zhao and D. Liu, Bioresour. Technol., 2015, 182, 136.

4 Y. Zeng, S. Zhao, S. Yang and S. Ding, Curr. Opin. Biotechnol., 2014, 27, 38-45.

5 S. Ding, Y. Liu, Y. Zeng, M. E. Himmel, J. O. Baker and E. A. Bayer, Science, 2012, 338, 1055.

6 M. Hall, P. Bansal, J. H. Lee, M. J. Realff and A. S. Bommarius, FEBS J., 2010, 277, 1571.

7 M. Cui, Y. Zhang, R. Huang, R. Su, W. Qi and Z. He, RSC Adv., 2014, 4, 44659.

8 M. Yoshida, Y. Liu, S. Uchida, K. Kawarada, Y. Ukagami, H. Ichinose, S. Kaneko and K. Fukuda, Biosci., Biotechnol., Biochem., 2008, 72, 805.

9 J. S. Kim, Y. Y. Lee and T. H. Kim, Bioresour. Technol., 2016, 199, 42.

10 R. Singh, A. Shukla, S. Tiwari and M. Srivastava, Renewable Sustainable Energy Rev., 2014, 32, 713.

11 X. Zhao, K. Cheng and D. Liu, Appl. Microbiol. Biotechnol., 2009, 82, 815 . 
12 J. Y. Zhu, X. J. Pan, G. S. Wang and R. Gleisner, Bioresour. Technol., 2009, 100, 2411.

13 J. Y. Zhu, M. S. Chandra, F. Gu, R. Gleisner, R. Reiner, J. Sessions, G. Marrs, J. Gao and D. Anderson, Bioresour. Technol., 2015, 179, 390.

14 Z. Zhang, M. D. Harrison, D. W. Rackemann, W. O. S. Doherty and I. M. O'Hara, Green Chem., 2016, 18, 360.

15 X. J. Pan and Y. Sano, Holzforschung, 1999, 53, 511.

16 X. Zhao and D. Liu, Bioresour. Technol., 2012, 117, 25.

17 H. Chen, J. Zhao, T. Hu, X. Zhao and D. Liu, Appl. Energy, 2015, 150, 224.

18 R. Wu, X. Zhao and D. Liu, ACS Sustainable Chem. Eng., 2016, 4, 1255-1261.

19 R. Sindhu, P. Binod, K. Satyanagalakshmi, K. U. Janu, K. V. Sajna, N. Kurien, R. K. Sukumaran and A. Pandey, Appl. Biochem. Biotechnol., 2010, 162, 2313.

20 N. Sathitsuksanoh, Z. Zhu, S. Wi and Y. H. P. Zhang, Biotechnol. Bioeng., 2011, 108, 521.

21 N. Sathitsuksanoh, Z. Zhu and Y. H. P. Zhang, Bioresour. Technol., 2012, 117, 228-233.

22 Y. Zhang, J. B. Cui, L. R. Lynd and L. R. Kuang, Biomacromolecules, 2006, 7, 644-648.

23 N. Sathitsuksanoh, A. George and Y. H. P. Zhang, J. Chem. Technol. Biotechnol., 2013, 88, 169.

24 Y. P. Zhang, S. Ding, J. R. Mielenz, J. Cui, R. T. Elander, M. Laser, M. E. Himmel, J. R. McMillan and L. R. Lynd, Biotechnol. Bioeng., 2007, 97, 214.

25 A. A. Elgharbawy, M. Z. Alam, M. Moniruzzaman and M. Goto, Biochem. Eng. J., 2016, 109, 252.

26 X. Zhao, L. Zhang and D. Liu, Biofuels, Bioprod. Biorefin., 2012, 6, 561.

27 M. Abe and H. Ohno, Production of biofuels and chemicals with ionic liquids, Springer, Netherlands, 2014.

28 M. Shafiei, K. Karimi and M. J. Taherzadeh, Bioresour. Technol., 2010, 101, 4914.

29 H. Zhang, J. Wu, J. Zhang and J. S. He, Macromolecules, 2005, 38, 8272 .

30 C. Kuo and C. Lee, Bioresour. Technol., 2009, 100, 866.

31 X. Tian, Z. Fang, D. Jiang and X. Sun, Biotechnol. Biofuels, 2011, 4, 53.

32 A. Sluiter, B. Hames, R. Ruiz, C. Scarlata and J. Sluiter, Laboratory Analytical Procedure, National Renewable Energy Laboratory, 2008, NREL/TP-510-42618.

33 X. Zhao, L. Zhang and D. Liu, Biotechnol. J., 2010, 5, 493.
34 X. Zhao, Y. Song and D. Liu, Enzyme Microb. Technol., 2011, 49, 413.

35 A. D. French and M. S. Cintron, Cellulose, 2013, 20, 58.

36 Q. Wang, Z. Wang, F. Shen, J. Hu, F. Sun, L. Lin, G. Yang, Y. Zhang and S. Deng, Bioresour. Technol., 2014, 166, 420.

37 A. A. Modenbach and S. E. Nokes, Biomass Bioenergy, 2013, 56, 526.

38 J. B. Kristensen, L. G. Thygesen, C. Felby, H. Jorgensen and T. Elder, Biotechnol. Biofuels, 2008, 1, 5.

39 B. Davison, J. Parks, M. Davis and B. Donohoe, in, Aqueous Pretreatment of Plant Biomass for Biological and Chemical Conversion to Fuels and Chemicals, ed. C. Wyman, John Wiley \& Sons, Ltd, Chichester, UK, 2013, pp. 23-38.

40 X. Zhao and D. Liu, BioEnergy Res., 2013, 6, 436-447.

41 H. Li, Y. Pu, R. Kumar, A. J. Ragauskas and C. E. Wyman, Biotechnol. Bioeng., 2014, 111, 485-492.

42 X. Zhao, L. Zhang and D. Liu, Biotechnol. J., 2010, 5, 493-504. 43 S. Y. Oh, D. I. Yoo, Y. Shin and G. Seo, Carbohydr. Res., 2005, 340, 417.

44 A. Xu, X. Guo and R. Xu, Int. J. Biol. Macromol., 2015, 81, 1000.

45 S. Tang, G. A. Baker, S. Ravula, J. E. Jones and H. Zhao, Green Chem., 2012, 14, 2922.

46 H. Sun, J. Miao, Y. Yu and L. Zhang, Appl. Phys. A: Mater. Sci. Process., 2015, 119, 539.

47 S. Park, J. O. Baker, M. E. Himmel, P. A. Parilla and D. K. Johnson, Biotechnol. Biofuels, 2010, 3, 10.

48 M. Wada, M. Ike and K. Tokuyasu, Polym. Degrad. Stab., 2010, 95, 543.

49 J. B. Binder and R. T. Raines, Proc. Natl. Acad. Sci. U. S. A., 2010, 107, 4516-4521.

50 H. Wang, G. Gurau and R. D. Rogers, Chem. Soc. Rev., 2012, 41, 1519-1537.

51 J. Hong, X. Ye and Y. H. P. Zhang, Langmuir, 2007, 23, 12535.

52 T. H. Kim and T. H. Kim, Energy, 2014, 66, 13-19.

53 G. Liu, J. Zhang and J. Bao, Bioprocess Biosyst. Eng., 2016, 39, 133.

54 F. H. Isikgor and C. R. Becer, Polym. Chem., 2015, 6, 4497.

55 J. Lange, E. van der Heide, J. van Buijtenen and R. Price, ChemSusChem, 2012, 5, 150.

56 Y. H. P. Zhang, J. Ind. Microbiol. Biotechnol., 2008, 35, 367.

57 J. Kautto, M. J. Realff, A. J. Ragauskas and T. Kassi, BioResources, 2014, 9, 6041.

58 W. Yang, P. Li, D. Bo and H. Chang, Carbohydr. Res., 2012, $357,53$. 